Gestão e Desenvolvimento, 21 (2013), 123-146

\title{
DESENHAR E IMPLEMENTAR UM SISTEMA DE LEARNING ANALYTICS NO ENSINO SUPERIOR
}

\author{
Sérgio André Ferreira* \\ António Andrade ${ }^{\dagger}$
}

\begin{abstract}
Resumo: A utilização de plataformas tecnológicas com base de funcionamento online, com destaque para os Learning Content Management System (LCMS), tem ganho uma importância crescente nas Instituições de Ensino Superior (IES). Da atividade dos alunos e professores nestas plataformas resulta um imenso trilho de cliques, que se traduz no registo de um enorme volume de dados - Big Datano sistema. A ideia do Learning Analytics (LA) é simples e tem associado um potencial transformativo muito elevado: $o$ aproveitamento destes dados permite um processo de tomada de decisão mais informada, abrindo as portas a um novo modelo na gestão das IES nos campos pedagógico e da eficiência organizacional. Contudo, a abordagem à temática dos LA ainda está na infância e a operacionalização eficaz exige respostas a grandes desafios no domínio tecnológico, educacional e das políticas. $O$ trabalho aqui apresentado insere-se neste contexto. Na Universidade Católica Portuguesa - Porto está em curso o desenvolvimento de um sistema LA alimentado com dados do LCMS institucional Blackboard - que tem como objetivo posicionar cada unidade curricular (UC) e faculdade numa matriz de cinco níveis de integração do LCMS no processo formativo. A matriz foi construída com base em modelos internacionais e considerou-se as funcionalidades oferecidas pelo LCMS. Para dar resposta aos requisitos desta matriz, desenhou-se todo o backoffice do sistema de extração e análise de dados no LCMS. Adicionalmente, foi construída e validada uma escala que contempla as mesmas dimensões, para aferição da opinião dos estudantes sobre a integração $e$ a importância do LCMS no seu processo de ensino e aprendizagem. Depois de concluída a construção deste LA é objetivo articular esta informação com os resultados académicos dos estudantes (Sistema de Gestão Académica) e avaliação dos docentes/ disciplinas (SIGIQ) dando-se passos na construção de um Academic Analytics.
\end{abstract}

* CEDH , FEP, Centro Regional do Porto da Universidade Católica Portuguesa

${ }^{\dagger}$ CEGE, FEG, Centro Regional do Porto da Universidade Católica Portuguesa 
Palavras-chave: Learning Content Management System, Learning Analytics

\begin{abstract}
The use of technology platforms based on online operating, amongst which we can highlight the Learning Content Management System (LCMS), has gained increasing importance in Higher Education Institutions (HEIs). From the activity of students and teachers on these platforms results a huge trail of clicks which is translated into a huge volume of data - Big Data - in the system. The idea of Learning Analytics (LA) is simple and is associated with a very high potential to transform: the use of this data may allow a more informed process of making decisions, opening the door to a new model in the management of HEIs in the fields of teaching and organizational efficiency. However, the approach to the theme of $L A$ is in its infancy and its effective operationalization requires responses to major challenges in technology, education and policies. This study fits into this context. At Universidade Católica Portuguesa - Porto there is an ongoing development of an LA system fed with data from the institutional LCMS - Blackboard which aims to position each course (UC) and faculty in a matrix with five levels of integration of LCMS in the teaching and learning process. The matrix was built based on international models and the functionality offered by LCMS was taken into consideration. To meet the requirements of this matrix, all the backoffice of the system of data extraction and analysis in the LCMS was drawn up. Additionally, we developed and validated a scale that covers the same dimensions, to gauge students' opinions on the importance and integration of the LCMS in their process of teaching and learning. After completing the construction of this LA the goal is to articulate this information with the learning results of students (Academic Management System) and the evaluation of teachers / subjects (SIGIQ) - taking steps in building an Academic Analytics.
\end{abstract}

Keywords: Learning Content Management System, Learning Analytics

\title{
1. INTRODUÇÃO
}

No ocaso do milénio passado, na PC Magazine escreveu-se que o Google "tem a capacidade inesgotável de devolver resultados [de pesquisa] extremamente relevantes" (Google, 2013). As sugestões de compras que empresas como a Amazon ou a eBay enviam para o e-mail dos clientes ou as que aparecem indicadas na área de cliente dos respetivos websites apresentam produtos com potencial interesse muito elevado. Do mesmo modo, quando alguém lê um e-mail no Gmail, abrese uma barra lateral com publicidade, fazendo-se uma breve análise ao seu conteúdo, é fácil constatar que uma parte significativa dos anúncios se relaciona com o teor dos e-mails do cliente. Estes são três, entre $n$ 
exemplos, reveladores do conhecimento profundo que algumas empresas e serviços online têm sobre as nossas preferências e o que cada um de nós procura. A maior parte das pessoas já se deparou com a dificuldade em escolher um livro para ler nas férias ou o presente certo para um amigo próximo. Contudo, estes dilemas parecem ser um não-assunto para as empresas e serviços online. Baseadas em informação deixada pelos nossos cliques ao longo dos trilhos do espaço virtual, apresentam uma série de sugestões, que, porventura, revelam um conhecimento muito maior sobre o que procuramos e os nossos gostos do que um amigo próximo - estamos no campus do business intelligence. Thomas Davenport "argues that business intelligence should be divided into querying, reporting, OLAP, an "alerts" tool, and business analytics. In this definition, business analytics is the subset of BI based on statistics, prediction, and optimization" (Wikipedia, n.d. Henschen \& Davenport, 2010).

$\mathrm{O}$ aproveitamento das vantagens da tecnologia, nomeadamente o armazenamento e análise de dados - incluindo modelos de previsão permite, através da definição de algoritmos complexos, extrair informação útil de conjuntos muito grandes de dados. Os três casos apresentados nas linhas anteriores são exemplos do potencial da aplicação prática dos grandes volumes de dados que cada um de nós deixa registado sempre que entra no mundo virtual. $\mathrm{O}$ uso do analytics em áreas como a Gestão e o Marketing tem já uma longa tradição: "Data-driven decision making, popularized in the 1980s and 1990s, is evolving into a vastly more sophisticated concept known as big data that relies on software approaches generally referred to as analytics"(Picciano, 2012, p. 9).

Existe uma relação simbiótica entre a crescente facilidade, e consequente universalidade, no uso da Tecnologia da Informação (TI) e a capacidade que a tecnologia tem "compreender" os seres humanos, no sentido de dar significado a grandes volumes de dados que indicam preferências, identificam padrões e traçam perfis: "Everything that human beings are doing to make it easier to operate computer networks is at same time, but for different reasons, making it easier for computer networks to operate human beings" (Dyson, 1998, location:267-84).

Se na Gestão e em áreas afins, o analytics já está numa fase de maturidade, o seu uso na Educação ainda está na infância. Dois fatores importantes concorrem para este estádio embrionário: i) a manifestação mais tardia do interesse pelo potencial do analytics pelos atores do setor da Educação; ii) a exigência da conjunção de requisitos de ordem 
tecnológica, educacional e organizacional exponencia a complexidade (e dificuldade) no desenvolvimento de sistemas de analytics.

Apesar da situação complexa da aplicação dos analytics na Educação, esta é uma temática claramente emergente, prenunciando-se a sua adoção consolidada num futuro próximo pelas IES - segundo o Horizon Reports (Johnson et al., 2013), a dois ou três anos.

O trabalho que aqui se apresenta tem dois propósitos: i) problematizar as principais questões discutidas no campo do Learning Analytics (LA), que, devido à novidade e potencial importância nos sistemas de Educação, justifica a divulgação e sistematização do conhecimento produzido - por aqui se fundamenta a pertinência teórica do trabalho; ii) descrever o processo de implementação de um LA numa instituição do ensino superior (IES), a Católica - Porto, que tem como objetivo posicionar cada unidade curricular (UC), faculdade/ escola e Centro Regional do Porto numa matriz com cinco níveis de integração do LCMS no processo de ensino e aprendizagem, construída com base na revisão da literatura.

O LA agrega dados provenientes de duas fontes: i) os relatórios do LCMS. Para dar resposta a este ponto foi desenvolvido um trabalho de backoffice para adequar os dados dos relatórios do LCMS à matriz de posicionamento; ii) opiniões dos estudantes sobre o uso e a importância do LCMS na sua atividade formativa. Para o efeito foi construído e validado a escala para o efeito.

Para além desta introdução, este artigo contempla mais cinco secções. Na secção dois é apresentada a revisão da literatura sobre assuntos relevantes dentro da temática LA; na secção três é caracterizada a dinâmica do LCMS da Católica - Porto que justifica a implementação de um LA; na seção quatro, "notas metodológicas sobre o desenho do LA", são fundamentados os propósitos do LA e as opções metodológicas; na seç̧ão cinco são apresentados os principais resultados alcançados; na secção seis são expostas as conclusões e indicados trabalhos futuros.

\section{LEARNING ANALYTICS}

\subsection{Dando sentido aos big data numa perspetiva holística e multidisciplinar}

Vannevar Bush no seu artigo As We May Think, publicado na revista The Atlantic Monthly, em 1945, problematizou a questão da gestão da informação num quadro em que se verifica o seu crescimento exponencial: "but rather that publication has been extended far beyond 
our present ability to make real use of the record" (Bush, 1945). Bush temia que o progresso científico fosse limitado pela incapacidade dos cientistas se manterem atualizados sobre informação relevante para o seu trabalho.

David L. M. Levy (2005), decorridos sessenta anos da publicação do As We May Think, revisita o artigo fazendo uma análise crítica, onde argumenta que, apesar das massivas inovações tecnológicas, a sobrecarga de informação não só se mantém como se exacerbou, sendo fundamental criar condições para existir tempo e espaço para reflexão sobre ela: "This paper revisits the article in light of current technological and social trends. It notes that Bush's argument centered around the problem of information overload and observes that in the intervening years, despite massive technological innovation, the problem has only become more extreme. It goes on to argue that today's manifestation of information overload will require not just better management of information but the creation of space and time for thinking and reflection, an objective that is consonant with Bush's original aims."(Levy, 2005, p. 281).

O relatório Policy Brief - Learning Analytics, da UNESCO, também realça a importância de dar sentido aos Big Data, tendo como suporte a infraestrutura tecnológica, mas colocando essencial da questão na reflexão e em novas formas de pensamento para o aproveitamento do potencial dos dados: "However, technology alone is just part of the story: appropriately skilled analysts are needed to make sense of the data, in order to inform decision-making (...) The term Big Data is used to reflect that a quantitative shift of this magnitude is in fact a qualitative shift demanding new ways of thinking, and new kinds of human and technical infrastructure. This raises a host of opportunities and challenges for society at large, and for institutions seeking to make sense of this data" (Shum, 2012, p. 2).

A questão essencial do LA converge com a temática de reflexão de Bush (1945) e Levy (2005): potenciar o aproveitamento de um imenso volume de dados, Big Data, na gestão dos ambientes de aprendizagem. O processo formativo no ensino superior com algum tipo de suporte online é um segmento em rápida expansão, visível não só nos cursos $e$-learning e b-learning, mas, também, nos cursos com regimes de frequência mais tradicionais - presencial a tempo completo. Da atividade online resulta uma imensa quantidade de dados que ainda não são bem explorados, mas que que podem ser preciosos na informação e compreensão sobre o processo de ensino e aprendizagem. Quando os estudantes utilizam o espaço virtual para aprender deixam dados com registo sobre os caminhos 
trilhados, que contêm informação sobre vários aspetos como, por exemplo, as suas preferências de aprendizagem ou as ferramentas utilizadas. Agregar estes dados em larga escala pode revelar padrões de aprendizagem (Siemens \& Gasevic, 2012).

$\mathrm{Na}$ linha do diagnóstico feito Bush há mais de seis décadas atrás, o crescimento dos dados ameaça a capacidade das organizações para lhes dar sentido (Swan, 2012). De facto, a temática do LA é complexa, agregadora de numerosos domínios, cobrindo todo o espectro entre as disciplinas de cariz mais tecnológico e os campos da teoria social da aprendizagem, onde se incluem disciplinas como a Ciência da Computação, Sociologia, Ciências da Educação, Estatística e Big Data (G. Siemens \& Gasevic, 2012). Utilizando a metáfora do espectro da luz, se se aplicasse o prisma de Newton ao LA, seria possível ver a sua decomposição por as várias disciplinas (figura I). Na perspetiva de Siemens e Gasevic: "The literature indicate LA as a field with potential for improving teaching and learning. Less clear, currently, is the longterm trajectory of LA as a discipline" $(2012$, p. 1).

\section{Figura I}

A transdisciplinaridade do LA

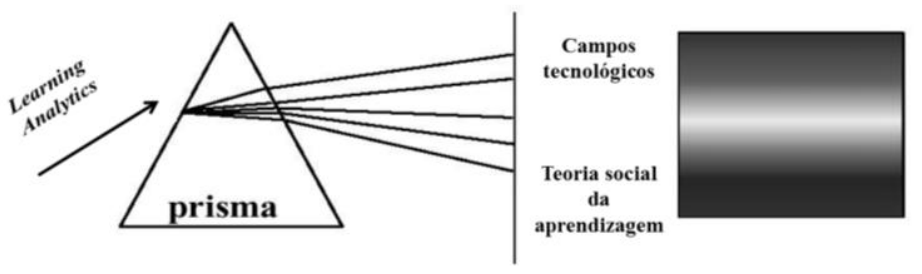

A encruzilhada do LA entre o campo tecnológico e o campo social requer, por um lado, um trabalho muito especializado ao nível programação de algoritmos e personalização de modelos; por outro, a capacidade dos atores das áreas de Gestão e das Ciências da Educação em definir as suas necessidades de informação e conseguir compreender e potenciar o uso dos algoritmos na transformação da realidade (Siemens \& Gasevic, 2012). A LAK12 sobre a temática dos LA foi apresentada como uma conferência "multidisciplinary conference for: learning scientists, (computer) scientists and data/knowledge engineers; (...), researchers in education, sociology, psychology, information science, (educators at all levels, (...) course management system developers and leaders." (LAK12, 2012). Na mesma linha, Siemens e Gasevic escrevem "Coalescing as a 
field will require leadership, openness, collaboration, and a willingness for researchers to approach learning analytics as a holistic process that includes both technical and social domains" (Siemens \& Gasevic2012, p. $1)$.

\subsection{Analytics e o processo de tomada de decisão nos sistemas de educação a várias escalas}

A Society for Learning Analytics Research (SoLAR) apresenta uma definição de LA em que covergem todas as vertentes desta problemática: "Learning analytics is defined as the measurement, collection, analysis and reporting of data about learners and their contexts, for purposes of understanding and optimising learning and the environments in which it occurs" (SoLAR, 2012).

A definição de LA da SoLAR tem implícita a seguinte assunção: o LA faz uso de dados preexistentes, que podem ter uma leitura em sistemas informáticos e que pelas suas propriedades são incompatíveis com uma leitura fora do ambiente tecnológico (Ferguson, 2012).

A figura II sintetiza os aspetos fundamentais da arquitetura de uma infraestrutura tecnológica de suporte ao LA, que incluem o armazenamento de dados, uma forma de pesquisa (data mining) e um modo de seleção (desagregação de dados). $\mathrm{O}$ armazenamento de dados refere-se a uma base ou múltiplos sistemas de base de dados que têm a valência de armazenar, integrar e manter grandes quantidades de dados ao longo do tempo. No LA é necessário garantir o acesso às diferentes fontes de dados, contudo, isso não significa que as bases de dados tenham de estar sob responsabilidade do mesmo serviço ou instituição, podendo ser bases de dados externos às quais se tem privilégios de acesso. A seleção de dados refere-se à capacidade de efetuar pesquisas para conseguir determinado tipo de dados que permita compreender um fenómeno específico. A desagregação dos dados refere-se ao uso de ferramentas informáticas para permitir a leitura dos dados sob diversos ângulos de análise. Por exemplo, selecionar dados para aferir o desempenho dos alunos por sexo, grupo ou outras características definíveis (Picciano, 2012). Resumidamente, pode-se dizer que o objetivo dos LA é a recolha e análise da informação para otimizar e prever (Henschen \& Davenport, 2010). 


\section{Figura II}

Arquitetura da infraestrutura tecnológica do LA

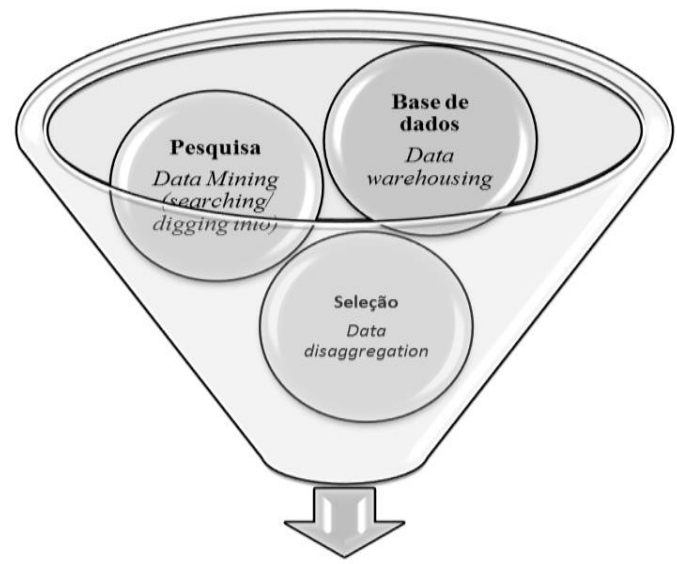

Tomada de decisão

Data-driven decision making

Se a arquitetura da infraestrutura tecnológica de suporte aos LA tem características comuns, a escolha do público-alvo e a definição dos objetivos condiciona diferentes formas de conceptualização dos problemas e modos de realização da recolha e apresentação dos dados (Ferguson, 2012). O LA compreende um raio de aplicação muito alargado, podendo centrar-se num nível macro, como políticas internacionais e nacionais, neste caso servem essencialmente os governos e ao nível institucional e supra-institucional. O nível meso responde à escala institucional e tem o focus nas questões de estratégia. Siemens (2011), Long e Siemens (2011), Shum (2012) fazem parte de um grupo alargado de autores que distinguem LA de Academic Analytics, sendo que este último conceito está mais relacionado com o nível institucional (nível meso) e supra-institucional (nível macro), associando-se ao conceito de Business Intelligence. No nível meso, o Academic Analytics visa orquestrar dados com um mix administrativo e LA para informar os administradores e o marketing da instituição. A microescala do LA suporta o rastreamento e interpretação da atividade do aluno - e por extensão dos grupos, cursos, departamentos, faculdade - , pelo que fornece dados com uma granularidade muito fina que serve aos próprios alunos, os professores e órgão da faculdade. Este nível dos LA é o mais personalizável e dependendo das plataformas pode mostrar os dados da atividade dos alunos online click-by-click, geolocalização, interações 
entre estudantes. Segundo Long e Siemens "learning analytics centers on the learning process (which includes analyzing the relationship between learner, content, institution, and educator)" (Long \& Siemens, 2011, p. 36)

\subsection{Desenvolvimento e uso pelas IES: desafio técnico e educacional}

O uso dos LA pelas IES levará alguns anos para amadurecer, contudo, a sua presença já se faz sentir e não deve ser ignorada (Picciano, 2012). No desenvolvimento e uso dos LA, as IES enfrentam dois desafios principais: o desafio tecnológico e o desafio educacional.

O desafio tecnológico relaciona-se com a capacidade das IES desenvolver aplicações informáticas que permitam aprofundar modos de extração, agregação e visualização de dados e elaboração de relatórios constituindo-os em Analytics. Na perspetiva de Dawson (2009) e Ferguson (2012) estas valências, fundamentais para o LA, ainda estão numa fase muito embrionária: "Although student tracking capabilities are typically included as generic software features, the depth of extraction and aggregation, reporting and visualisation functionality of these built-in analytics has often been basic or non-existent" (Ferguson, 2012, p. 4). Outra vertente do desafio tecnológico que se coloca tem origem na situação de uma significativa parte da atividade online do aluno ter lugar em múltiplas plataformas, muitas das quais integrando o seu PLE, mas fora da alçada institucional: "In addition, significant amounts of learner activity take place externally, and so records are distributed across a variety of different sites with different standards, owners and levels of access" (Ferguson, 2012, p. 4). Ferguson (2012) sintetiza a problemática do desafio técnico na adoção dos LA: "The first driver, then, is a technical challenge: How can we extract value from these big sets of learning-related data?" (p. 4).

$\mathrm{O}$ desafio educacional refere-se à capacidade dos atores das IES conseguir otimizar o uso da informação na gestão dos ambientes de aprendizagem ricos em tecnologia e potenciar a eficácia do processo de ensino aprendizagem: "The second driver is therefore na educational challenge: How can we optimise opportunities for online learning? (Ferguson, 2012, p. 4).

O New Media Consortium apresenta anualmente um relatório, The Horizon Report, sobre as tendências relacionadas com a tecnologia, que vão marcar a evolução no modo como as IES se organizam e como desenvolvem o processo de ensino e aprendizagem. A edição de 2011 do 
Horizon Report (Johnson, Smith, Willis, Levine, \& Haywood, 2011) estimava um período de quatro a cinco anos para adoção do LA pelas IES, sendo que a edição de 2012 (Johnson, Adams, \& Cummins, 2012) antecipa este horizonte temporal para um período de dois a três anos. Brown (2011) enumera três fatores que impulsionam a emergência do LA: i) O LA disponibiliza informação útil e atualizada sobre o que alunos fazem ao longo do curso. Esta informação é considerada cada vez mais fundamental para a gestão da atividade formativa em tempo real; ii) Até muito recentemente, se uma IES queria utilizar o LA tinha de o desenvolver de raiz, o que se revelava um processo moroso e com obstáculos técnicos consideráveis. Atualmente, marcas como a SunGard (SunGard, 2012), Blackboard (Blackboard, 2012) e Desire2Learn (Desire2Learn, 2012) começaram a dar passos nesta direção, fator que pode acelerar a adoção dos LA; iii) Crescente utilização de métricas no ensino superior, no sentido de demonstrar aspetos variados da atividade formativa da instituição. Nesta linha, existem vários projetos que envolvem IES de todo o mundo sobre a temática dos LA (Educause, 2010) e sistemas de deteção e gestão da atividade ou presença online dos indivíduos (MIT, 2011).

$\mathrm{Na}$ literatura existem grandes expectativas sobre o potencial do LA em fornecer novos contributos nos processos de tomadas de decisão, nas práticas educativas e formas de melhorar o ensino e a aprendizagem. A sua concretização e adequação têm sidos assuntos em discussão na literatura neste campo ainda emergente, mas em rápido crescimento (Siemens \& Gasevic, 2012).

\section{DINÂMICA DO USO DO LCMS NA CATÓLICA - PORTO}

A Católica - Porto é atualmente um ambiente rico em tecnologia (TELE - Technology Enhanced Learning Environment). Na figura III apresenta-se as principais cenas do filme da construção do TELE institucional, onde o campus online, o LCMS - Blackboard, assume um papel de relevo.

O ano letivo de 1997/98 a instituição atribuiu uma conta de e-mail e disponibilizou um website pessoal, para os docentes colocarem os conteúdos das unidades curriculares.

No ano letivo 2003/2004 iniciou-se a exploração do Training/Teaching Web Toolkit (TWT) para suporte atividade letiva. Esta plataforma permite a criação e exploração de ambientes de ensino e aprendizagem baseados na internet. O número de UC suportadas pelo TWT aumentou em dois anos de 13 para 254. 
Em 2003/2004 os centros regionais de Lisboa e Porto lançaram a primeira edição do Mestrado em Informática Educacional em regime de e-learning. Este mestrado atualmente tem a oitava edição em funcionamento e é um exemplo de pioneirismo e longevidade neste tipo de cursos. Ao longo das suas oito edições, este mestrado captou estudantes de todo território continental e regiões autónomas. A plataforma de suporte ao funcionamento do curso foi a Netforma no primeiro meio ano, que depois substituída pela Blackboard até à atualidade.

No ano letivo 2005/ 2006, iniciou-se um período experimental com o Moodle no ensino presencial, com o objetivo de mostrar uma nova tendência alternativa ao TWT. Posteriormente, a Blackboard é escolhida para plataforma institucional substituindo o Moodle. Inicia-se o processo de generalização da utilização da Blackboard em todas as UC presenciais oferecidas pela instituição e no aproveitamento do potencial da plataforma no desenvolvimento de cursos de e-learning (Mestrado em Gestão e Organização Escolar, da Faculdade de Educação e Psicologia), dois resultantes de parcerias internacionais (Mestrado em Integração Económica Regional e Políticas Comunitária, em parceria com a Universidade de Vigo e um Doutoramento em conjunto com a Universidade Católica de Moçambique).

\section{Figura III}

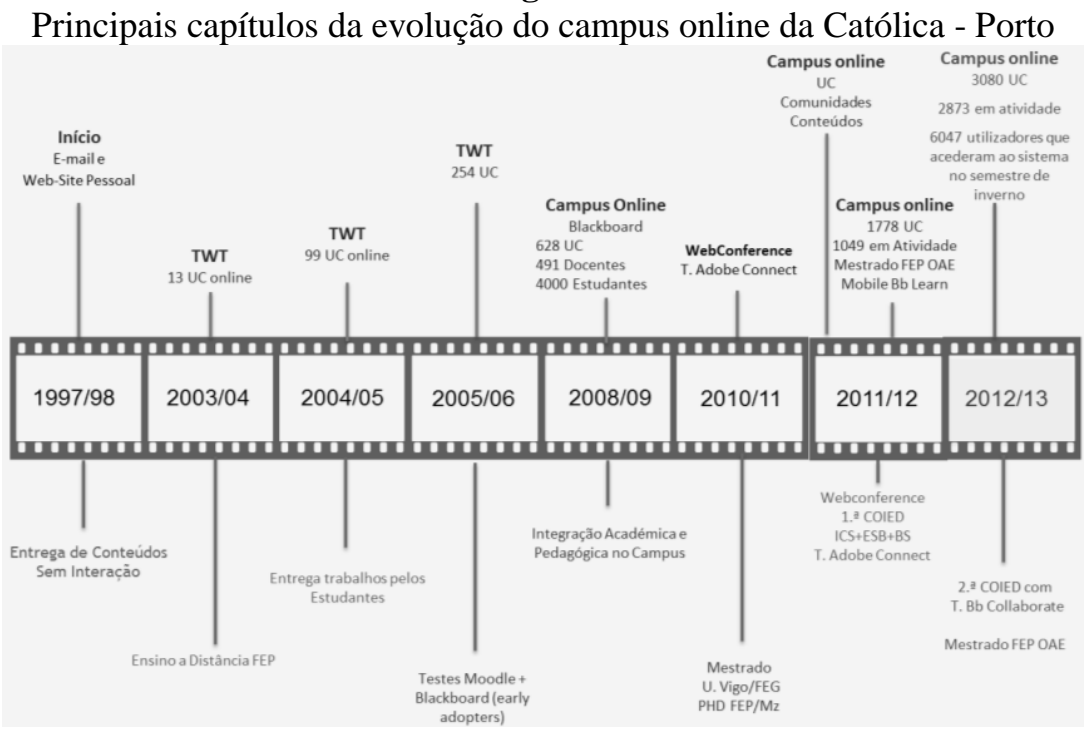


No ano letivo 2010/ 2011 fez-se a integração da plataforma de Webconference Adobe Connect e em 2012/ 2013 da Blackboard Colloborate, o que tem permitido complementar a Blackboard no apoio à lecionação, desenvolvimento de cursos e organização de conferências web.

Na Figura IV é apresentada a dinâmica da utilização do LCMS no semestre de inverno de 2012/2013 por utilizadores registados e pelo público. No semestre de inverno (1 setembro - 31 dezembro), o campus registou perto de 300 mil visitas, com prevalência de origem distrito do Porto, mas com valores importantes de todo o território nacional e uma fração de visitantes fora de Portugal. O tempo médio por visita foi 7 minutos e o número de páginas vistas/ visita situou-se nas $16 \%$. Um indicador importante, e a ser seguido em termos de futuro, é o acesso via mobile que já tem uma quota de $10 \%$, o que aprece alinhado com tendências emergentes no campo da Educação, em concreto, do mobile learning. O volume de UC criadas (3080) e o número de utilizadores registados que acederam (6047) revelam uma utilização importante do campus.

\section{Figura IV}

Dinâmica da utilização do LCMS no semestre de inverno de 2012/2013 por utilizadores registados e pelo público

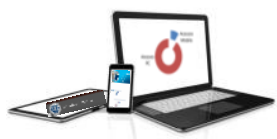

- 294055 visitas

- Tempo médio/ visita: minutos

- 16 páginas vistas e1 cada entrada

- $10 \%$ dos acessos são vi mobile
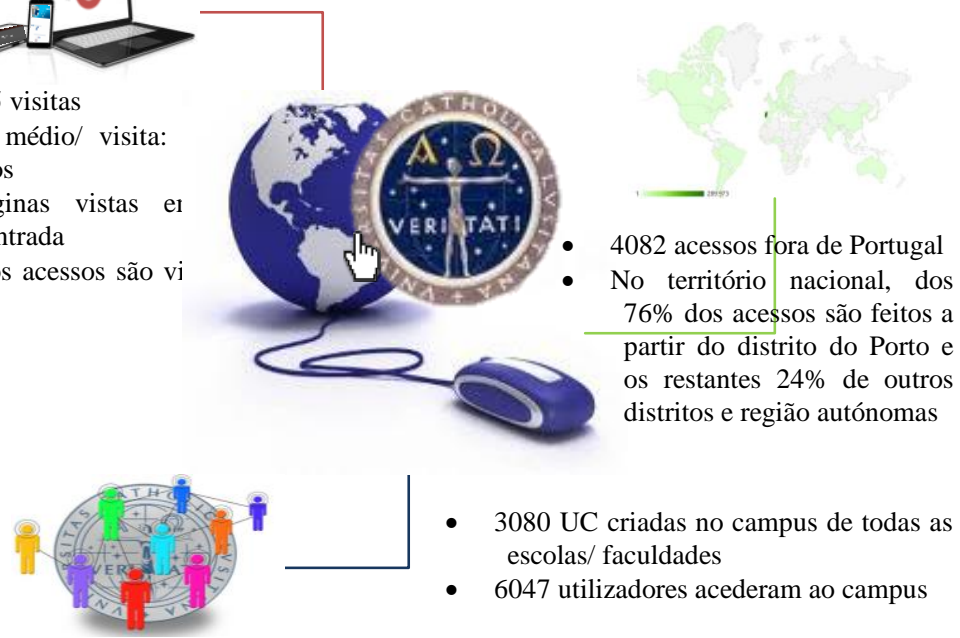

- 3080 UC criadas no campus de todas as escolas/ faculdades

- 6047 utilizadores acederam ao campus 
Numa sondagem realizada na plataforma após login foi colocada uma sequência de perguntas aos estudantes sobre aspetos relacionados coma integração da Blackboard na sua aprendizagem. As questões são adaptadas do modelo de Kaczynski, Wood e Harding (2008) às quais que responderam cerca 300 estudantes. As respostas dos estudantes corroboram os dados dos relatórios da Blackboard.

$\mathrm{Na}$ questão "Qual a dinâmica de acesso ao Campus para ter sucesso no curso?", 67\% dos estudantes afirmou ser necessário aceder diariamente à Blackboard e $95 \%$ referiu ser necessário fazer pelo menos 2 a 3 acessos por semana (figura $\mathrm{V}$ ).

\section{Figura V}

Qual a dinâmica de acesso ao campus para ter sucesso no curso?

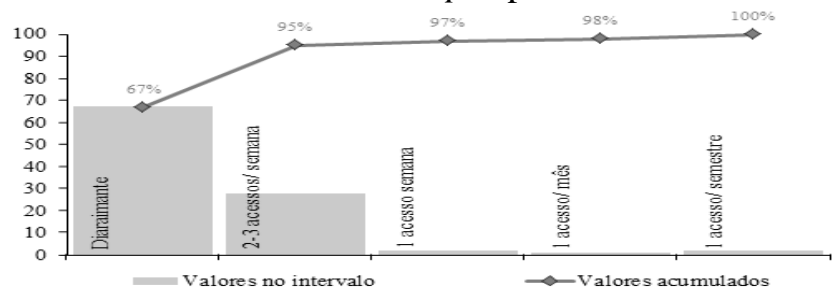

Relativamente à questão "Que fração das informações/ conteúdos relevantes para as disciplinas do curso que frequento é disponibilizada na Blackboard?", $62 \%$ dos respondentes declarou ser mais de metade (figura $\mathrm{VI})$.

\section{Figura VI}

Que fração das informações/ conteúdos relevantes para as disciplinas do curso que frequento é disponibilizada na Blackboard?

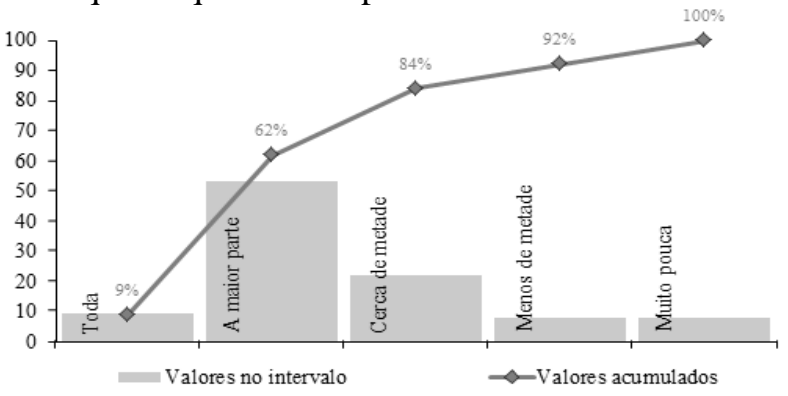

$\mathrm{Na}$ questão "Que fração da avaliação (fóruns, blogues, testes, entrega trabalhos), com implicações na classificação final, é feita no Campus 
online?", $28 \%$ dos estudantes declara ser realizada cerca de metade ou mais (figura VII).

\section{Figura VII}

Que fração da avaliação (fóruns, blogues, testes, entrega trabalhos), com implicações na classificação final, é feita no campus online?

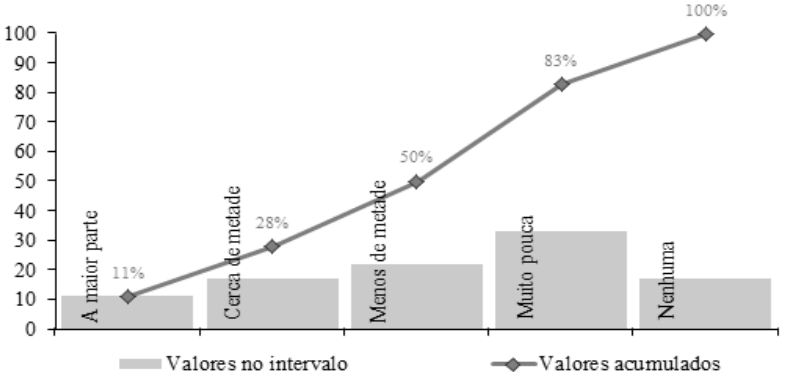

A dinâmica do uso da Blackboard é, assim, demonstrada pela evolução histórica, pelos relatórios do sistema e pela opinião dos estudantes. Por aqui se justifica, o investimento no desenvolvimento de um sistema de LA que permita compreender os impactos, posicionando as diversas estruturas da Católica - Porto em níveis de integração do LCMS no processo formativo.

\section{NOTAS METODOLÓGICAS SOBRE O DESENHO DO LA}

Três fatores principais contribuíram para a ideia de desenhar e implementar um sistema de LA na Católica - Porto:

- O investimento da instituição num TELE, cuja vertente mais visível é o LCMS. Esta plataforma tecnológica tem um uso generalizado nas UC, importando perceber qual a integração efetiva nas várias dimensões do processo de ensino e aprendizagem. $\mathrm{O}$ objetivo passa não só por gerir melhor, mas também pela utilização da informação para favorecer uma adaptação rápida a novas situações.

- A Católica - Porto tem em funcionamento um Sistema de Garantia e Avaliação da Qualidade Interna (SIGIQ), contudo, a sua ação não contempla uma avaliação específica de aspetos relacionados com a utilização da tecnologia no processo de ensino e aprendizagem, em particular do LCMS, que se assume uma peça central no TELE institucional. A literatura recomenda que se definam critérios específicos para avaliação da introdução da tecnologia no ensino e aprendizagem, de modo a facilitar a transição para o novo paradigma: "Management has to focus on transition, be proactive and serve as a 
role model, since changes in technology often produce chaotic situations. Furthermore, the institution's internal quality assessment model for teaching and learning has to be expanded to include criteria specific to e-learning" (SNAHE, 2008, p. 52). Este projeto insere-se neste contexto, estando prevista a articulação do LA com o trabalho do SIGIQ, dando-se passos na construção de um Academic Analytics.

- O LA é referido na literatura como uma tendência emergente e com grande potencial na transformação do ensino superior: "Basing decisions on data and evidence seems stunningly obvious, and indeed, research indicates that data-driven decision making improves organizational output and productivity" (Long \& Siemens, 2011, p. 31) A aposta e o comprometimento da Católica - Porto na utilização da tecnologia para potenciar as práticas pedagógicas, demonstrado ao longo do seu historial (figura III), é um incentivo e justifica que se trilhe este caminho.

Estes fatores encerram questões de ordem organizacional, educacional e tecnológica. O genoma do sucesso do LA passa pela resposta às dimensões deste triângulo:

- Dimensão organizacional - Espera-se que o LA dê resposta aos requisitos de informação da instituição, fornecendo informação útil sobre a integração do LCMS no processo de ensino e aprendizagem, que possa ser agregada por múltiplos níveis de análise que traduzam a organização da instituição: universidade, faculdade/ escola e UC. A organização da informação, por níveis de detalhe, possibilita o acesso condicionado à informação, se esse for o objetivo da instituição. Nesse caso, cada professor só terá acesso à informação sobre as UC que leciona, o diretor de faculdade/escola a todas a UC da instituição que dirige, os Serviços de Gestão da Qualidade (SIGIQ) e a direção da Católica - Porto a toda a informação.

- Dimensão educacional - Na literatura são múltiplos os modelos que caraterizam a evolução da integração da tecnologia na atividade formativa online, apresentando descritores para fazer este posicionamento (e.g. Technology Florida Center for Instructional , 2011). Com base na revisão da literatura e nas funcionalidades oferecidas pelo LCMS, foram definidas as dimensões do modelo de análise (figura IX).

- Dimensão tecnológica- O desenho de todo o backoffice do sistema de extração e análise de dados foi realizado para dar resposta aos requisitos das dimensões organizacional e educacional, mas exigindo uma conciliação constante com a exequibilidade tecnológica. Num 
processo dialético foram feitas progressivas aproximações de modo a garantir-se que o LA reflita uma gestão equilibrada das três dimensões do triângulo.

Com base na Technology Integration Matrix, do Florida Center for Instructional (2011), desenvolveu-se um instrumento de posicionamento das UC e das Escolas/Faculdades em cinco níveis: introdução, adoção, adaptação, imersão e transformação. Na figura VIII apresenta-se as descrições genéricas dos cinco estádios (Ferreira \& Andrade, 2012).

\section{Figura VIII}

Níveis de integração do LCMS no processo de ensino aprendizagem

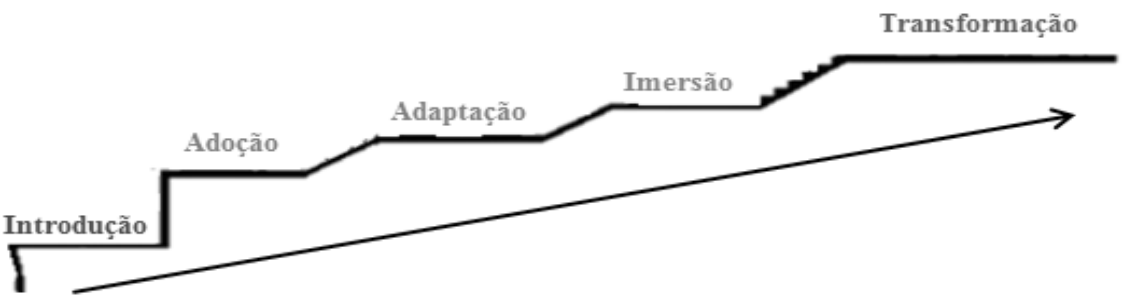

Introdução - É possível ter sucesso à UC sem aceder ao LCMS.

Adoção - O LCMS tem um impacto limitado, mas visível, no processo de ensino e aprendizagem. $\mathrm{O}$ estudante sente dificuldades em ter sucesso à UC sem aceder ao LCMS.

Adaptação - É extremamente difícil ao estudante ter sucesso na UC sem aceder ao LCMS.

Imersão - O LCMS tem um grande impacto no processo de ensino e aprendizagem. O estudante não consegue ter sucesso na UC sem aceder ao LCMS.

Transformação - O LCMS é vital e tem um poder transformador no processo de ensino e aprendizagem $\mathrm{O}$ estudante não consegue ter sucesso na UC sem aceder ao LCMS.

\section{Figura IX}

Dimensões para avaliação da integração do LCMS na prática letiva

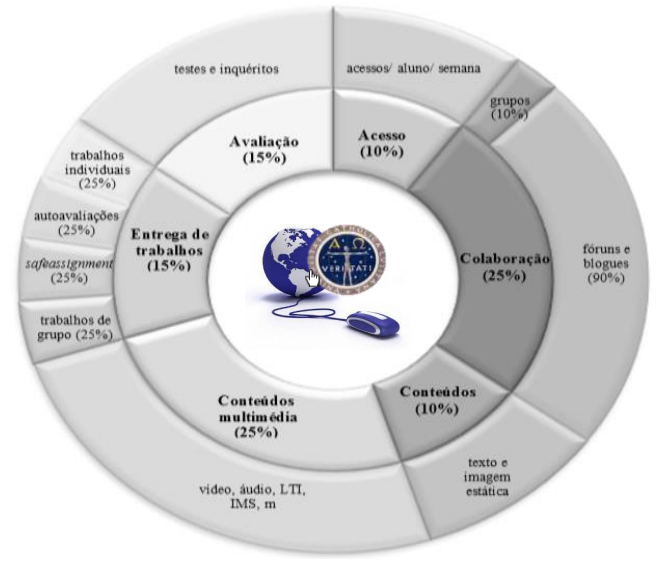


$\mathrm{Na}$ figura IX estão representadas as dimensões consideradas no modelo, que refletem dimensões que percorrem as principais valências oferecidas pelo LCMS e, cumulativamente, consideram dimensões identificadas na literatura como fundamentais para o desenvolvimento das atividades letivas no ambiente online, a saber: Acesso, colaboração, conteúdos, conteúdos multimédia, entrega de trabalhos e avaliação. Estas dimensões têm diferentes pesos no cálculo do posicionamento global da UC, consoante a preponderância que assumem no processo de ensino e aprendizagem (Ferreira \& Andrade, 2012).

Para além dos dados dos relatórios do Blackboard, pretende-se aferir a perceção dos alunos sobre o grau de integração do LCMS no seu processo de aprendizagem, nesse sentido foi desenvolvida uma escala com as mesmas seis dimensões presentes nos relatórios do LCMS, à qual foi adicionada a dimensão comunicação, que não consta dos relatórios da Blackboard, devido a questões de exequibilidade tecnológica, pois o sistema não permite a contagem dos itens desta dimensão. Desta forma, não foi possível integrar esta dimensão no backoffice dos relatórios do LCMS.

\section{RESULTADOS}

Os resultados já alcançados traduziram-se na concretização de um sistema de análise e extração de dados do LCMS - produto do desenho realizado em backoffice - e no desenvolvimento e validação de uma escala sobre a integração do campus no processo de ensino e aprendizagem. Na figura $\mathrm{X}$ apresenta-se o relatório da UC Sistemas de Informação e Tecnológicos, da Faculdade de Economia e Gestão, da Católica - Porto, em que se concretiza o desenho das figuras VIII e IX. Neste seguimento, para cada dimensão é encontrado o estádio de posicionamento, calculado considerando os seguintes pontos de maturação: introdução [0-20\%[; adoção [20\%-40\%[; adaptação [40\%60\%[; imersão [60\%-80\%[; transformação[80\%-100\%[. 


\section{Figura $\mathbf{X}$}

Dimensões para avaliação da integração do LCMS na prática letiva

\begin{tabular}{|c|c|c|c|c|c|}
\hline Dimensões & Estágio & $\begin{array}{l}\text { Ponto de Maturação } \\
(\%)\end{array}$ & Item & Total & $\begin{array}{l}\text { Média } \\
\text { p/semana }\end{array}$ \\
\hline Acessos & Introdução & 1 & Acessos & 323 & 0,07 \\
\hline \multirow{3}{*}{ Colaboração } & \multirow{3}{*}{ Transformação } & \multirow{3}{*}{100} & Grupos & 69 & $\mathrm{~N} / \mathrm{A}$ \\
\hline & & & Fónuns & 7 & $\mathrm{~N} / \mathrm{A}$ \\
\hline & & & Blogs & 0 & $\mathrm{~N} / \mathrm{A}$ \\
\hline \multirow{4}{*}{$\begin{array}{l}\text { Entrega de } \\
\text { Trabalhos }\end{array}$} & \multirow{4}{*}{ Adaptação } & \multirow{4}{*}{50} & Trabalhos & 4 & $\mathrm{~N} / \mathrm{A}$ \\
\hline & & & Autoavaliações & 0 & N/A \\
\hline & & & SafeAssign & 0 & $\mathrm{~N} / \mathrm{A}$ \\
\hline & & & $\begin{array}{l}\text { Trabalhos de } \\
\text { Grupo }\end{array}$ & 60 & $\mathrm{~N} / \mathrm{A}$ \\
\hline Conteúdos & Transformação & 100 & Conteúdos & 59 & 3,93 \\
\hline \multirow{6}{*}{$\begin{array}{l}\text { Conteúdos } \\
\text { Multimédia }\end{array}$} & \multirow{6}{*}{ Adaptação } & \multirow{6}{*}{50} & Videos & 2 & $\mathrm{~N} / \mathrm{A}$ \\
\hline & & & Áudio & 0 & $\mathrm{~N} / \mathrm{A}$ \\
\hline & & & Scorm & 0 & $\mathrm{~N} / \mathrm{A}$ \\
\hline & & & LTI & 0 & $\mathrm{~N} / \mathrm{A}$ \\
\hline & & & IMS & 0 & $\mathrm{~N} / \mathrm{A}$ \\
\hline & & & Mashups & 3 & $\mathrm{~N} / \mathrm{A}$ \\
\hline \multirow{2}{*}{ Avaliações } & \multirow{2}{*}{ Transformação } & \multirow{2}{*}{100} & Testes & 20 & $\mathrm{~N} / \mathrm{A}$ \\
\hline & & & Inquénitos & 1 & $\mathrm{~N} / \mathrm{A}$ \\
\hline
\end{tabular}

Os pontos de maturação de cada dimensão podem ser parametrizados de modo flexível, de acordo com os objetivos da instituição. Na figura XI observa-se o backoffice para parametrização do estádio adaptação, existindo tantas tabelas de parametrização, quantos os estádios do modelo - no caso cinco. Para cada item é necessário definir-se os valores mínimos e máximos, sendo que o ponto de maturação de cada dimensão é determinado por um algoritmo em que é feita a ponderação dos itens que a constituem.

\section{Figura XI}

Ponderação dos itens para determinação dos estádios O exemplo do estádio adaptação

3. Adaptaçăo
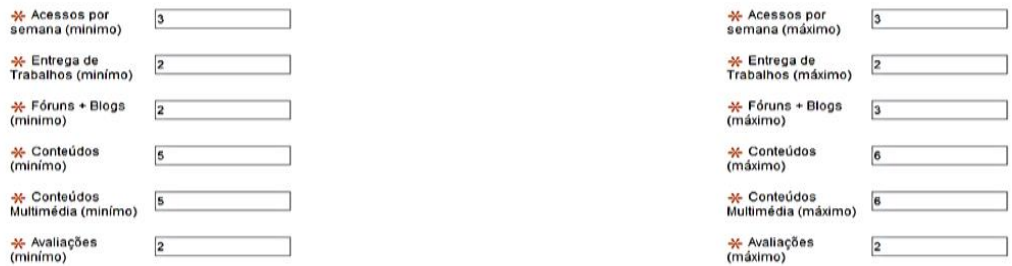
Do mesmo modo, a partir dos resultados dos pontos de maturação das diferentes dimensões (figura X), é calculado o estádio global da UC. O posicionamento da UC no estádio é efetuado através de um algoritmo que reflete as ponderações atribuídas a cada uma das dimensões (figura IX):

$\sum=($ acesso $* 0,1)+($ colaboração $* 0,25)+($ conteúdos $* 0,1)+($ conteúdos multimédia*025)+(Entrega de trabalhos $* 0,15 \%)+($ avaliação*0,15).

Na figura XII pode observar-se o posicionamento global da UC Sistemas de Informação e Tecnológicos no estádio imersão (cf, figura VIII), que reflete este procedimento.

\section{Figura XII}

Posicionamento global da UC "Sistemas de Informação e Tecnológicos"

\begin{tabular}{|l|l|l|l|}
\hline \multicolumn{2}{|c|}{ Dados da Unidade Curricular } \\
Código da UC & Unidade Curricular & Estágio & $\begin{array}{l}\text { Ponto de } \\
\text { Maturação (\%) }\end{array}$ \\
\hline 201213_F614C1072S1 & $\begin{array}{l}\text { (FEG) SISTEMAS DE INFORMAÇÃO E } \\
\text { TECNOLOGICOS (Ano 2012/13 } 1^{\circ} \text { Semestre) }\end{array}$ & Imersão & 70 \\
\hline
\end{tabular}

A figura XIII apresenta um gráfico radar do sistema - também produto do desenho backoffice - que permite fazer uma leitura discriminada da integração do LCMS na UC nas diferentes dimensões. No caso da UC em análise, pode concluir-se que o posicionamento global no estádio imersão resulta do alto grau de integração das dimensões colaboração, conteúdos e avaliação (pontos de maturação=100\%) no processo de ensino e aprendizagem. Em contrapartida, o contributo das dimensões entrega de trabalhos e conteúdos multimédia é mais modesto, havendo um caminho a percorrer nestes campos (ponto de maturação $=50 \%$ ). Relativamente à dimensão dinâmica de acessos está num nível baixo, revelando um número de acessos/ alunos/ semana muito reduzido.

\section{Figura XIII}

Posicionamento da UC "Sistemas de Informação e Tecnológicos" nas diferentes dimensões avaliadas - dados do sistema

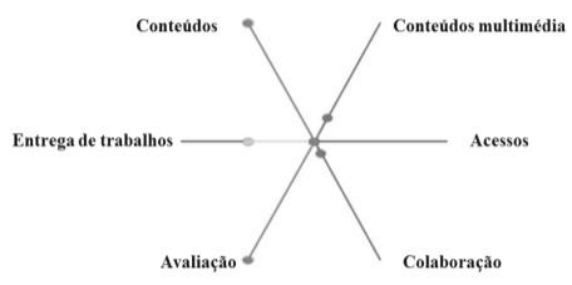


A leitura cruzada dos dados do sistema com a opinião dos estudantes -recolhida através da aplicação de uma escala sobre a integração e a importância das diferentes dimensões do LCMS no processo de ensino e aprendizagem - está presente na figura XIV, na forma de um gráfico radar.

\section{Figura XIV}

Posicionamento da UC "Sistemas de Informação e Tecnológicos" Cruzamento dos dados do sistema com a opinião dos utilizadores

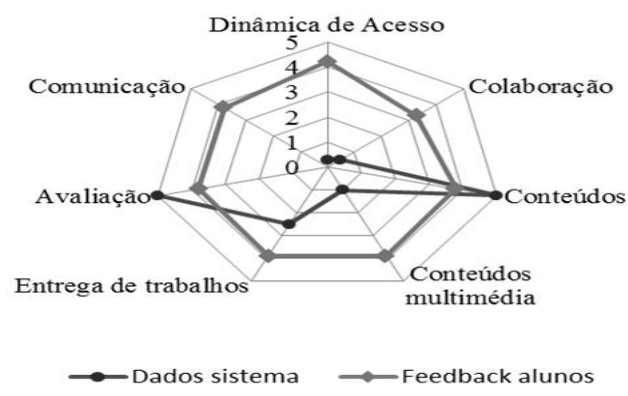

O momento atual ainda é de desenvolvimento e implementação do LA, contudo, já é possível dar uma perspetiva geral da integração do LCMS no processo de ensino e aprendizagem da Católica - Porto (Figura $\mathrm{XV}$ ), por faculdade. O principal facto a reter é que a dinâmica do uso do LCMS (figuras IV a VII) não tem correspondência níveis de exploração do campus (figura $\mathrm{XV}$ ). De facto, a figura $\mathrm{XV}$ evidencia que todas as faculdades têm a maioria das UC posicionadas no estádio introdução, sendo pouco expressivo o número de UC posicionadas em níveis superiores.

\section{Figura XV}

Níveis de exploração do Campus pelas diferentes Unidades Académicas no $1 .^{\circ}$ Semestre de 2012/13.

\begin{tabular}{|c|c|c|c|c|c|c|c|}
\hline Faculdade & Nivel Global & Nivel G & Introduçăo & Adoção & Adaptaçäo & Imersäo & Transformaçāo \\
\hline Faculdade de Economia e Gestão & Introdução & 6 & 328 & 10 & 1 & 1 & 0 \\
\hline Teologia & Introdução & 4 & 278 & 7 & o & o & o \\
\hline Instituto de Bioética & Introdução & 3 & 1 & o & o & 0 & o \\
\hline Instituto de Ciências da Saúde & Introdução & 5 & 379 & 9 & o & o & o \\
\hline Escola de Direito & Introdução & 4 & 408 & 3 & o & o & o \\
\hline Business Scholl & Introdução & 4 & 450 & o & o & o & o \\
\hline Escola Superior de Biotecnologia & Introdução & 5 & 345 & 5 & o & o & o \\
\hline Faculdade de Educação e Psicologia & Introdução & 5 & 274 & 10 & 1 & o & o \\
\hline Governação e Políticas Públicas & Introdução & 4 & 22 & 0 & o & 0 & $\circ$ \\
\hline Escola das Artes & Introdução & 3 & 477 & 3 & 1 & o & o \\
\hline Mais Saber & Introdução & 3 & 66 & 1 & o & o & o \\
\hline Totais & 3080 & & 3028 & 48 & 3 & 1 & 0 \\
\hline
\end{tabular}




\section{CONCLUSÕES E TRABALHOS FUTUROS}

O trabalho aqui apresentado teve dois contributos principais: i) colocou em equação o quadro teórico e as aplicações práticas dos sistemas de Learning e Academic Analytics, uma temática emergente com grande potencial para o aumento da eficiência na condução de políticas e práticas educativas, a várias escalas de análise; ii) concretizou o desenho de uma arquitetura de um sistema de LA que tem como focus de análise a integração do campus da Católica - Porto, o LCMS Blackboard, no processo formativo.

O projeto de LA apresentado neste trabalho foi registado nos Horizons Projects (NMC, 2012), sendo classificado com níveis 5 nos campos Interestingness e Relevance, numa escala de mínimo 1 e máximo 5, o que é um indicador da sua pertinência.

As dimensões consideradas no sistema de LA - acesso, colaboração, conteúdos, conteúdos multimédia, entrega de trabalhos e avaliação - têm correspondência nas funcionalidades do campus e são sustentadas na literatura. O backoffice dos relatórios do LCMS desenhado permite o posicionamento de cada UC numa matriz de cinco níveis - introdução, adoção, adaptação, imersão e transformação - inspirada na "Technology Integration Matrix" (Technology Florida Center for Instructional, 2011) . Os resultados do semestre de inverno de 2012/ 2013 indicam que a maioria das UC e das faculdades se encontram no estádio introdução, o que corresponde a um nível de exploração baixo das funcionalidades do campus. Todavia, a dinâmica de uso do campus (figuras IV a VII) apresenta valores que revelam uma grande implementação nas atividades letivas. Fazendo uma leitura de conjunto das UC individuais, a ideia que emerge é que a Blackboard funciona essencialmente como um repositório de conteúdos, sendo a exploração das funcionalidades de colaboração, entrega de trabalhos, avaliação e a disponibilização de conteúdos multimédia menos efetiva, estando a sua exploração limitada a um reduzido de UC mais dinâmicas. Está a ser trabalhada uma solução que permita agregar os dados discriminados das diferentes dimensões por faculdades, o que possibilitará aferir ao nível da faculdade e da Católica Porto quais as dimensões com maior integração e menor integração.

Os dados do sistema de LA podem ser agregados por níveis hierárquicos que refletem o plano organizacional da Católica - Porto (UC, faculdade, centro regional). Estão em curso trabalhos sobre formas de integração dos dados da escala da opinião dos estudantes sobre a 
importância e integração do campus no processo formativo com os relatórios do LCMS (figura XIV).

A curto prazo, pretende-se articular a informação do LA apresentado neste trabalho com os resultados académicos dos estudantes (Sistema de Gestão Académica) e avaliação dos docentes/ disciplinas (SIGIQ) caminhando-se para a construção de um Academic Analytics (figuraXVI).

\section{Figura XVI}

Arquitetura de um possível Academic Analytics para a Católica - Porto

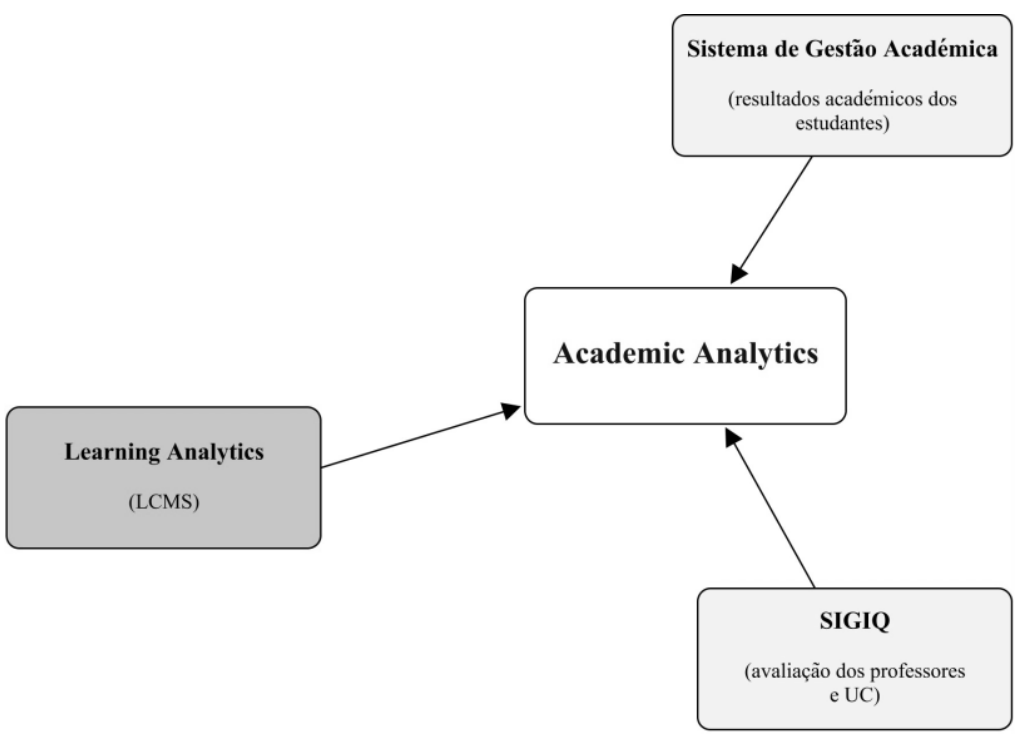

\section{BIBLIOGRAFIA}

Blackboard. (2012). Blackboard Analytics - Gain a Whole New Perspective Retrieved Jul 28, 2012, from http://www.blackboard.com/platforms/analytics/overview.aspx

Brown, M. (2011). Learning Analytics: The Coming Third Wave. EDUCAUSE Learning Initiative Brief. 1-4. Retrieved from doi:ELIB1101

Bush, V. (1945, July). As We May Think. The Atlantic, 281-286.

Dawson, S. (2009). 'Seeing' the learning community: an exploration of the development of a resource for monitoring online student 
networking. British Journal of Educational Technology, 41(5), 736-752.

Desire2Learn. (2012). Desire2Learn Analytics - Desire2Learn Analytics Retrieved Jul 28, 2012, from http://www.desire2learn.com/products/analytics/

Dyson, G. (1998). Darwin Among the Machines (Kindle DX ed.): Penguin Books.

Educause. (2010). Next Generation Learning Challenges: Learner Analytics Premises. Retrieved from Educause.edu website: $\mathrm{http}: / / \mathrm{w} w \mathrm{w}$.educause.edu/library/resources/next-generationlearning-challenges-learner-analytics-premises

Ferguson, R. (2012). The State Of Learning Analytics in 2012: A Review andFuture Challenges. Technical Report KMI-12-01. Milton Keynes, UK: Knowledge Media Institute, The Open University.

Ferreira, S. A., \& Andrade, A. (2012). Ambientes de aprendizagem ricos em tecnologia - arquitetura e contributos para a gestão. Revista Portuguesa de Investigação Educacional, 12, 241-272.

Google. (2013). Our history in depth Retrieved 02-04, 2013, from http://www.google.com/about/company/history/

Henschen, D., \& Davenport, T. (2010, 01-04). Analytics at Work: Q\&A with Tom Davenport Retrieved 03-12, 2013, from http://www.informationweek.com/software/business-

intelligence/analytics-at-work-qa-with-tom-davenport/222200096

Johnson, L., Adams Becker, S., Cummins, M., Estrada, V., Freeman, A., \& Ludgate, H. (2013). NMC Horizon Report: 2013 Higher Education Edition. Austin, Texas.

Johnson, L., Adams, S., \& Cummins, M. (2012). Horizon Report:2012 Higher Education Edition. Texas, Austin: The New Media Consortium.

Johnson, L., Smith, L. R., Willis, H., Levine, A., \& Haywood, K. (2011). The 2011 Horizon Report. Austin, Texas.

Kaczynski, D., Wood, L., \& Harding, L. (2008). Using radar charts with qualitative evaluation: Techniques to assess change in blended learning. Active Learning in Higher Education, 9(1), 23-41.

LAK12. (2012). Learning Analytics and Knowledge - LAK2012, from http://lak12.sites.olt.ubc.ca/

Levy, D. M. (2005). To Grow in Wisdom: Vannevar Bush, Information Overload, and the Life of Leisure. Paper presented at the JCDL '05 Proceedings of the 5th ACM/IEEE, Denver, Colorado, USA. 
Long, P., \& Siemens, G. (2011). Penetrating the fog: analycs in learning and education. Educause Review Online, (46), 31-40. Retrieved from http://net.educause.edu/ir/library/pdf/erm1 151.pdf

MIT. (2011). Personas: How does the Internet see you? Retrieved 08-28, 2012, from http://personas.media.mit.edu/

NMC. (2012). Horizon Project Navigator - Technology, Innovation and Education Retrieved 01-16, 2013, from http://navigator.nmc.org/project/technology-innovation-andeducation

Picciano, A. G. (2012). The Evolution of Big Data and Learning Analytics in American Higher Education. Journal of Asynchronous Learning Networks, 16(3), 9-20.

Shum, S. B. (2012). Policiy Brief - Learning Analytics. Moscow, Russian Federation: UNESCO.

Siemens, G. (2011). Learning and Academic Analytics Retrieved 01-28, 2012, from http://www.learninganalytics.net/?p=131

Siemens, G., \& Gasevic, D. (2012). Guest Editorial - Learning and Knowledge Analytics. Educational Technology \& Society, 15(3), $1-2$.

Snahe. (2008). E-learning quality: Aspects and criteria for evaluation of e-learning in higher education. Luntmakargatan.

Solar. (2012). Society for Learning Analytics Research Retrieved 01-16, 2013, from http://www.solaresearch.org/mission/about/

Sungard. (2012). SunGard Solutions Retrieved Jul 28, 2012, from http://www.sungard.com/astec

Swan, K. (2012). Introduction to the Special Issue on Learning Analytics. Journal of Asynchronous Learning Networks, 16(3), 5-7.

Technology Florida Center for Instructional -Technology Integration Matrix. (2011). from http://fcit.usf.edu/matrix/matrix.php

Wikipedia (s.d). Business intelligence. In Wikipedia. Retrieved 14 May, 2013, from http://en.wikipedia.org/wiki/Business_intelligence\#cite_note-8 